\title{
Hybrid-array-based optoacoustic and ultrasound (OPUS) imaging of biological tissues
}

\author{
Deán-Ben, X L ; Merčep, E ; Razansky, D
}

DOI: https://doi.org/10.1063/1.4983462

Posted at the Zurich Open Repository and Archive, University of Zurich ZORA URL: https://doi.org/10.5167/uzh-172702

Journal Article

Published Version

Originally published at:

Deán-Ben, X L; Merčep, E; Razansky, D (2017). Hybrid-array-based optoacoustic and ultrasound (OPUS) imaging of biological tissues. Applied Physics Letters, 110(20):203703.

DOI: https://doi.org/10.1063/1.4983462 


\section{Hybrid-array-based optoacoustic and ultrasound (OPUS) imaging of biological tissues}

Cite as: Appl. Phys. Lett. 110, 203703 (2017); https://doi.org/10.1063/1.4983462

Submitted: 25 January 2017. Accepted: 02 May 2017. Published Online: 19 May 2017

X. L. Deán-Ben, E. Merčep, and D. Razansky
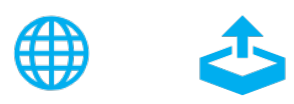

View Online

Export Citation

\section{ARTICLES YOU MAY BE INTERESTED IN}

Photoacoustic imaging in biomedicine

Review of Scientific Instruments 77, 041101 (2006); https://doi.org/10.1063/1.2195024

Microwave-excited ultrasound and thermoacoustic dual imaging

Applied Physics Letters 110, 183701 (2017); https://doi.org/10.1063/1.4983166

Four dimensional hybrid ultrasound and optoacoustic imaging via passive element optical excitation in a hand-held probe

Applied Physics Letters 105, 173505 (2014); https://doi.org/10.1063/1.4900520

\section{Applied Physics Letters}

Mid-IR and $\mathrm{THz}$ frequency combs special collection 


\title{
Hybrid-array-based optoacoustic and ultrasound (OPUS) imaging of biological tissues
}

\author{
X. L. Deán-Ben, ${ }^{1}$ E. Merčep, ${ }^{2,3}$ and D. Razansky ${ }^{1,2, a)}$ \\ ${ }^{1}$ Institute for Biological and Medical Imaging, Helmholtz Center Munich, Ingolstaedter Landstrasse 1, 85764 \\ Neuherberg, Germany \\ ${ }^{2}$ Faculty of Medicine, Technical University of Munich, Ismaningerstrasse 22, 81675 Munich, Germany \\ ${ }^{3}$ iThera Medical GmbH, Zielstattstrasse 13, 81379 Munich, Germany
}

(Received 25 January 2017; accepted 2 May 2017; published online 19 May 2017)

\begin{abstract}
Hybrid optoacoustic and pulse-echo ultrasound imaging is an attractive multi-modal combination owing to the highly complementary contrast of the two techniques. Efficient hybridization is often hampered by significant dissimilarities between their optimal data acquisition and image formation strategies. Herein, we introduce an approach for combined optoacoustic and ultrasound imaging based on a plano-concave detector array design with a non-uniform pitch distribution. The hybrid design optimized for both modalities allows for maintaining an extended field of view for efficient ultrasound navigation while simultaneously providing broad tomographic coverage for optimal optoacoustic imaging performance. Imaging sessions performed in tissue-mimicking phantoms and healthy volunteers demonstrate that the suggested approach renders an enhanced imaging performance as compared with the previously reported hybrid optoacoustic and ultrasound imaging approaches. Thus, it can greatly facilitate clinical translation of the optoacoustic imaging technology by means of its efficient combination with ultrasonography, a well-established clinical imaging modality. Published by AIP Publishing. [http://dx.doi.org/10.1063/1.4983462]
\end{abstract}

Optoacoustic (OA) and ultrasound (US) imaging share key advantages for biomedical applications such as the use of non-ionizing radiation, real-time imaging capability, high spatial resolution, and portable use. ${ }^{1}$ Efficient combination of the two modalities may lead to important advantages as US enables easy anatomical navigation and localization of structures, while OA can provide additional functional information, which translates into a better applicability in a clinical setting. As both the modalities are based on ultrasound detection, they can be, in principle, readily combined into a highly complementary hybrid imaging system. However, the conventional data acquisition and image formation strategies of B-mode ultrasound ${ }^{2}$ fundamentally differ from the tomographic approaches that render optimal optoacoustic image quality. ${ }^{3}$ This may often impose contradicting requirements when considering efficient hybridization of the two modalities.

B-mode US imaging is conventionally done with linear array transducers, enabling direct contact with the tissue in the hand-held operation mode. ${ }^{2}$ Standard image formation methods are based on steering the incident US beam and capturing the corresponding echoes over the desired field of view. The inter-element distance (pitch) of the array must preferably be kept below half the minimum acoustic wavelength in order to avoid the deterioration of image quality due to grating lobes and resolution loss. ${ }^{4}$ Inter-element isolation in ultrasound arrays must be carefully taken into account in the manufacturing process of the array to avoid cross-coupling between elements. ${ }^{5}$ On the other hand, large-area detection elements are typically employed in OA imaging systems in order to detect the generally weak and ultra-wideband responses induced by the transient absorption of pulsed laser radiation. ${ }^{3}$ Furthermore, the speckle-free nature of time-resolved OA

\footnotetext{
a) Author to whom correspondence should be addressed: dr@tum.de
}

signals commonly leads to a reduced visibility of structures when employing standard ultrasonography arrays. ${ }^{6}$ Indeed, OA imaging is inherently a tomographic modality that highly depends upon sufficient angular tomographic coverage for accurate image reconstruction. Thus, a concave array geometry is generally preferred in order to maximize the tomographic coverage around the imaged sample. ${ }^{7}$

Hybrid optoacoustic and ultrasound (OPUS) imaging was first achieved with linear arrays including optical illumination components that capitalize on the well-established structural imaging capacity of B-mode $\mathrm{US},{ }^{8,9}$ with multimodal imaging enabled using dedicated synchronization electronics. ${ }^{10,11}$ In order to overcome the generally unsatisfactory OA imaging performance obtained with linear and planar ultrasound arrays, especially designed concave arrays have been developed to achieve better image quality and quantification in hand-held (portable) OA imaging. ${ }^{1,12}$ One simple way of achieving simultaneous US imaging was by capturing echoes of US waves that were generated optoacoustically (passively) by illuminating optical absorbers placed outside the imaged sample. ${ }^{13}$ A similar approach was also implemented in the transmission mode for the speed of sound mapping in small animals. ${ }^{14,15}$ Alternatively, the elements of concave arrays can be actively driven with high voltage pulses for achieving conventional pulse-echo US imaging. ${ }^{16}$ However, the large angular aperture of the convex array geometries necessitated an extended number of individual elements for maintaining a reasonable interelement pitch and avoiding significant grating lobe artifacts in the US images while providing sufficient tomographic coverage for accurate OA imaging. ${ }^{17}$

Herein, we suggest an approach for hybrid OPUS imaging based on a multi-segment (plano-concave) detection array supporting optimal performance in both the modes 
(Imasonic SaS, Voray, France). Figure 1 shows the suggested configuration of the hybrid optoacoustic-ultrasound (OPUS) imaging probe consisting of a plano-concave array of cylindrically focused elements. While optimal pulse-echo US imaging performance is achieved with the central (linear) segment of the array, the OA responses are additionally collected by the two concave segments ensuring a broad tomographic coverage of $180^{\circ}$ in the OA imaging mode. The linear array segment consists of 128 piezocomposite elements with a $0.25 \mathrm{~mm}(1.25 \lambda)$ inter-element pitch, whereas each concave segment has 64 piezocomposite elements separated by a $0.6 \mathrm{~mm}(3 \lambda)$ pitch. The concave segments lie on a $40 \mathrm{~mm}$ radius arc, and all the elements are cylindrically focused at a distance of $38 \mathrm{~mm}$ within the imaging plane. The elements of the linear segment are focused at a distance of $34 \mathrm{~mm}$ from the active aperture. All the elements have at least $70 \%$ detection bandwidth around a central frequency of 7.5 MHz. OA excitation is provided using an optical parametric oscillator (OPO)-based laser (Innolas Laser $\mathrm{GmbH}$, Krailling, Germany) whose beam is guided through a custom-made fiber bundle (Ceramoptec $\mathrm{GmbH}$, Bonn, Germany). The output of the fiber bundle, located at the lateral side of the array, is oriented with an angle of $\sim 17^{\circ}$ so that the illumination beam intersects the cross-sectional imaging plane roughly at the tissue surface. The per-pulse fluence at the skin surface was estimated to be $12 \mathrm{~mJ} / \mathrm{cm}^{2}$, which conformed with the laser exposure safety standards. ${ }^{18}$

The US imaging protocol is defined as follows. The custom-made ultrasound imaging platform consolidates pulser-receiver boards and the function of triggered acquisition for synchronizing the US and OA data streams. ${ }^{16,17}$ The transmitted bi-polar US pulses at a frequency of $8 \mathrm{MHz}$ have a peak-to-peak voltage of $20 \mathrm{~V}$. The US images are reconstructed from the reflected signals detected by the linear segment of the array (128 channels) using a synthetic aperture technique ${ }^{19}$ in a field of view of $40 \times 40 \mathrm{~mm}^{2}$ with a pixel size of $0.18 \mathrm{~mm}$. The OA images are rendered using a tomographic reconstruction algorithm considering signals collected by all the 256 array elements. A field of view of $40 \times 40 \mathrm{~mm}^{2}$ with a pixel size of $0.1 \mathrm{~mm}$ was used. The OA signal acquisition was done using separate custom-made acquisition electronics that was synchronized to the laserinitiated trigger signal. Each laser pulse triggers the simultaneous acquisition of the generated optoacoustic signals by all the 256 transducer elements. Subsequently, each transducer
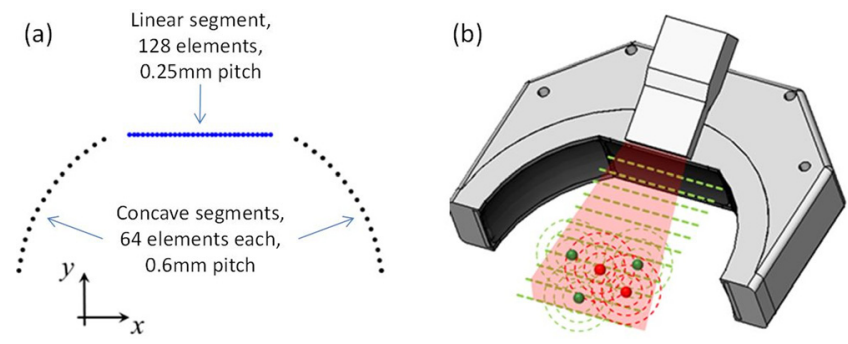

FIG. 1. Suggested plano-concave configuration of the hybrid optoacousticultrasound (OPUS) imaging probe. (a) Distribution of the array elements in the imaging plane. (b) 3D rendering of the hybrid imaging probe, including the illumination fiber bundle. element of the linear part of the array is sequentially excited and all the remaining elements collect the ultrasound echoes from the tissue. The imaging frame rate was determined by the pulse repetition frequency of the laser $(10 \mathrm{~Hz})$, and no signal averaging was performed.

Imaging performance of the hybrid array was first characterized in an agar phantom containing $\sim 95 \mu \mathrm{m}$ diameter polyethylene microspheres (Cospheric BKPMS 90-106, Santa Barbara, USA). For comparison, the same phantom was also imaged using a cylindrically focused concave array commonly employed for cross-sectional tomographic OA imaging that has a uniform curvature and an inter-element pitch. The concave array has very similar geometrical characteristics to the plano-concave array, i.e., the same radius of curvature of $40 \mathrm{~mm}$ and a total of 256 detection elements covering an angle of $180^{\circ}$ with an inter-element pitch of $0.47 \mathrm{~mm}(1.6 \lambda)$. All the elements have at least $70 \%$ detection bandwidth around a central frequency of $5 \mathrm{MHz}$. Figure 2 presents the phantom images acquired with the two arrays in both the OA and US modes. Figure 2(a) shows the US image obtained with the linear part of the plano-concave array having a small inter-element pitch. The particles and the upper and lower boundaries of the phantom are clearly visible, while no significant artefacts are present in the entire reconstructed field of view of $40 \times 40 \mathrm{~mm}^{2}$. On the other hand, the US image rendered by using all 256 elements of the planoconcave array [Fig. 2(b)] exhibits background artifacts, which are mainly attributed to the grating lobes generated by the concave parts of the array having a large inter-element pitch. Note that even stronger artifacts are observed in the US image obtained with the concave array having a large pitch between all its elements [Fig. 2(c)], which exhibits only a narrow effective field-of-view free of grating lobe artifacts. The corresponding OA images of the phantom are shown in Figs. 2(d)-2(f). Here, a standard back-projection algorithm was employed for the tomographic optoacoustic reconstructions. ${ }^{20}$ As expected, significant distortions of the particle shape are manifested in the OA image obtained with the linear array segment due to its limited angular coverage. On the other hand, the OA images obtained with all 256 elements of the plano-concave and concave arrays [Figs. 2(e) and 2(f)] have similar image quality. This is generally expected as both the arrays provide approximately the same angular coverage. Note that the inferior resolution for the concave array is due its lower detection bandwidth. Thus, it can be readily seen that the concave segments of the planoconcave array are mainly responsible for restoring the correct shape of the particles in the reconstructed images. It is also observed that the plano-concave design also achieves a larger effective field of view in the OA mode as compared with the concave array geometry [compare the areas with resolvable contrast from the particles shown in Figs. 2(e) and 2(f)], which represents an additional advantage of the suggested approach.

The spatial resolution of the hybrid imaging system was estimated in a separate experiment with a phantom containing a single $\sim 95 \mu \mathrm{m}$ polyethylene microsphere. Specifically, the particle was positioned in the center of the array and scanned along the elevational (z) direction for 10 positions with a step size of $200 \mu \mathrm{m}$. 100 signal averages were done 

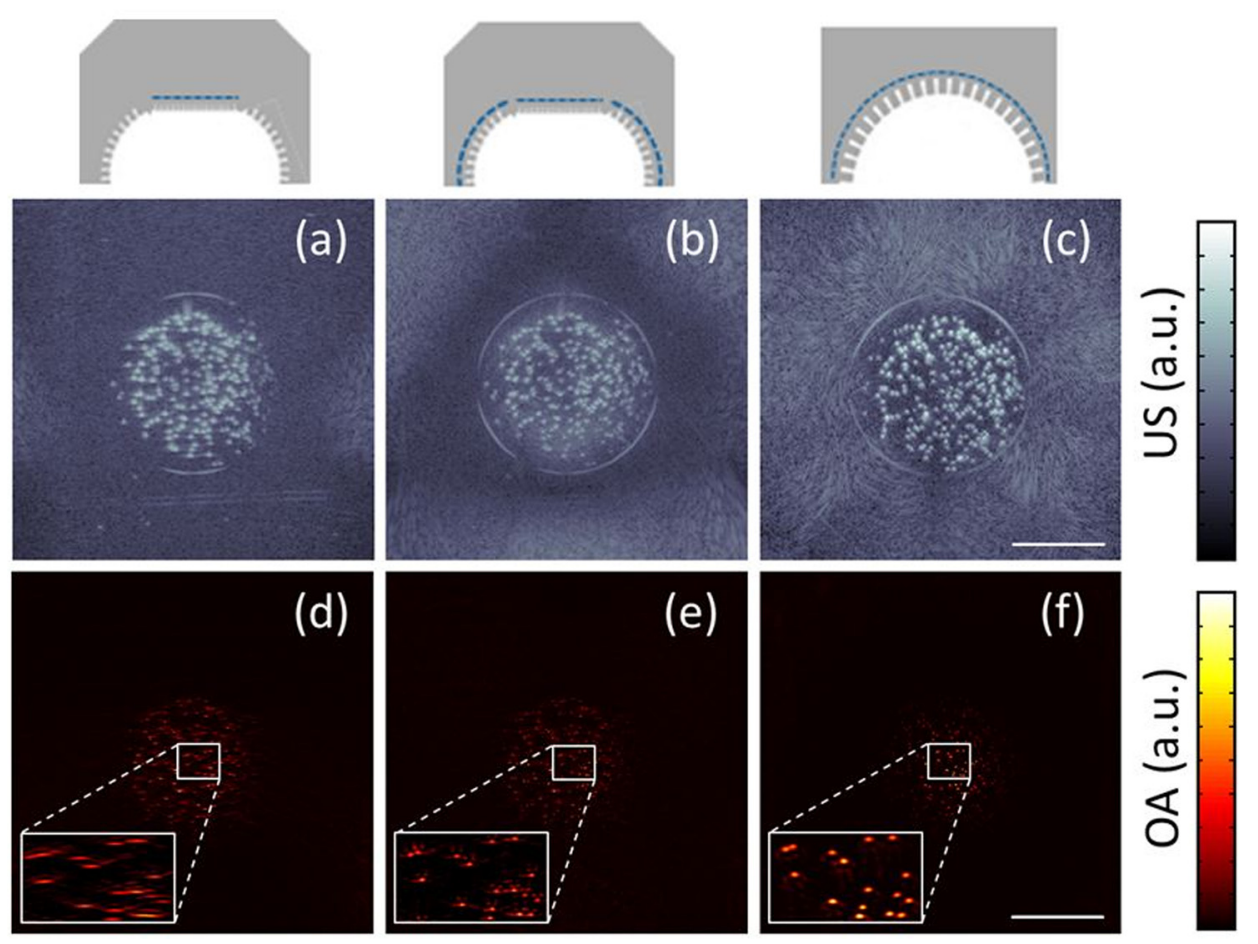

FIG. 2. Comparison of the US and OA images obtained with the different array configurations. (a) US image rendered with the linear part of the multisegment (hybrid) array. (b) Ultrasound image rendered with all the elements of the hybrid array. (c) Ultrasound image rendered with the concave array. (d) OA image rendered with the linear part of the hybrid array. (e) Optoacoustic image rendered with all the elements of the hybrid array. (f) Optoacoustic image rendered with the concave array. Scale bars $-10 \mathrm{~mm}$. for each scanning position. The out-of-plane resolution along the $\mathrm{z}$ direction was then estimated as the full width at half maximum (FWHM) of the reconstructed values for the scanned positions, resulting in approximate values of 0.9 and $1 \mathrm{~mm}$ for the OA and US scans, respectively. The in-plane resolution was finally estimated as the FWHM of the reconstructed cross-sectional images with the particle located at the center of the imaged plane, resulting in US and OA resolutions of $\sim 250 \mu \mathrm{m}$ and $\sim 110 \mu \mathrm{m}$, respectively.

In vivo imaging performance was subsequently tested by imaging a wrist of a healthy volunteer. The wavelength of the OPO laser was tuned to $800 \mathrm{~nm}$, and its pulse repetition frequency was kept at $25 \mathrm{~Hz}$. An example of the OA image obtained for a representative position of the wrist is shown in Fig. 3(a). In this case, the OA reconstruction was performed using an iterative model-based algorithm incorporating a non-negative constraint ${ }^{21}$ for better image quality. The corresponding US image is displayed in Fig. 3(b), while Fig. 3(c) shows a superposition of both the images. Some anatomical information, such as the skin surface, is visible in both the OA and US images. However, the two modalities appear to be highly synergistic with OA contrast mainly emphasizing the vasculature while US images providing highly complementary structural information on other soft tissues. The strong blood-related contrast of optoacoustics aids in clear
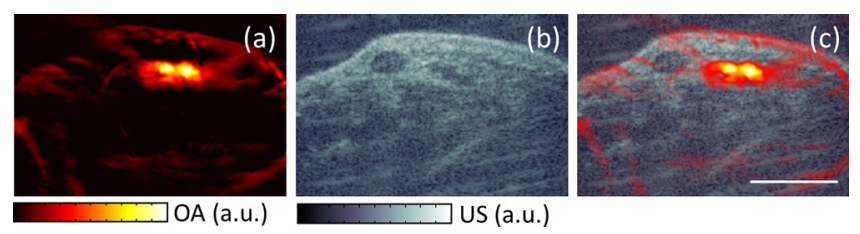

FIG. 3. Hybrid optoacoustic-ultrasound (OPUS) imaging in a wrist of a healthy volunteer. (a) OA image acquired at $800 \mathrm{~nm}$. (b) The corresponding US image captured simultaneously with the same probe. (c) A hybrid (OPUS) image. Scale bar- $10 \mathrm{~mm}$. differentiation of the vascular structures that may have a similar structural appearance to other anechoic structures (e.g., tendons) in the US images. Note that, as opposed to previously reported hybrid imaging systems based on linear arrays, ${ }^{8,9,22}$ good anatomical imaging performance was also achieved for the OA modality, which can be mainly attributed to the large tomographic coverage of the hybrid array. On the other hand, the US images rendered with the linear segment of the plano-concave array are equivalent to those obtained with the conventional B-mode arrays, making them easy to interpret by trained personnel.

The showcased results indicate the superior imaging performance of the suggested approach for hybrid OPUS imaging. The multi-segment design with a non-uniform pitch enables reducing the number of elements required for attaining optimal image quality, thus also reducing the complexity and cost of the data acquisition electronics. For instance, achieving similar US imaging performance with a standard concave geometry ${ }^{17}$ would necessitate at least double the number of elements if the pitch size is kept uniform. In addition, the linear segment with dense element distribution ensures artifact-free US imaging and a large field of view, the latter not achievable with a pure concave array design. The hybrid concept is thus poised to significantly accelerate the clinical acceptance of the OA technology. This is because the US images obtained with the linear part of the multi-segment array are equivalent to those rendered using standard US scanners, whereas the co-registered high quality OA images can add key functional information on blood perfusion and oxygenation, of great importance in many clinical diagnostic applications related to e.g., cardiovascular diseases, breast oncology, skin malignancies, arthritis, inflammation, or peripheral vascular diseases. ${ }^{23-27}$ The hybrid OPUS probe introduced herein is then poised to impact clinical applications by providing an otherwise unattainable combination between anatomical and functional information. 
Accurately registered OA and US images may provide an additional value if image formation is complemented with prior information provided by the other modality. For example, strong acoustic scatterers easily recognizable with pulseecho US are known to generate arc-type artefacts in OA images. ${ }^{28}$ Such deficiencies can be minimized if, e.g., the location of the acoustic scatterers is identified from the US images $^{29}$ or, alternatively, signals emitted by strong optical absorbers are emulated via proper excitation of the US array. ${ }^{30}$ The speckle reduction techniques commonly used for enhancing imaging performance in medical $\mathrm{US}^{31}$ may equally benefit from the high contrast information available in the OA images.

More generally, the proposed concept of hybrid OPUS imaging based on a non-uniform pitch and shape of the array segments optimized for both OA and US imaging can be further adapted to fit specific applications. Of particular relevance is the effective field of view, which is mainly affected by the size and the number of detection elements in OA imaging and by the number of acquired A-lines and beam steering parameters in standard US. The latter further depends on whether the array is curved, whereas convex arrays typically cover a larger field of view as compared with linear array geometries at the expense of inferior image quality. Thereby, a convex-concave array geometry may turn preferable in some cases of hybrid OPUS imaging. An array with a uniform (concave) curvature may also serve for hybrid imaging purposes if some of its segments are made suitable for pulse-echo US in terms of the inter-element pitch. Efficient hybrid OPUS imaging in three dimensions can also potentially be enabled by implementing the suggested multi-segment concept with hybrid matrix arrays, e.g., consisting of concave spherical parts optimized for 3D OA imaging combined with planar or convex segments tailored for 3D ultrasound. A similar strategy may similarly be implemented in other hybrid imaging systems, e.g., for endoscopic or whole-body small animal imaging applications.

In conclusion, the hybrid-array-based imaging approach introduced in this letter can greatly facilitate the clinical translation of the OA imaging technology by means of its efficient combination with the well-established US imaging modality. OA and US imaging are natural partners, both using information delivered by acoustic waves for image formation. Their efficient hybrid combination provides a clear added value as compared with standalone approaches, hence holding promise of becoming a major imaging tool in biomedical discovery and the clinics.

D.R. acknowledges funding from the European Research Council Consolidator Grant No. ERC-2015-CoG682379 .

\footnotetext{
${ }^{1}$ X. L. Deán-Ben and D. Razansky, "Functional optoacoustic human angiography with handheld video rate three dimensional scanner," Photoacoustics 1, 68-73 (2013).

${ }^{2}$ T. L. Szabo, Diagnostic Ultrasound Imaging: Inside Out (Academic Press, 2004).

${ }^{3}$ L. V. Wang, Photoacoustic Imaging and Spectroscopy (CRC press, 2009).

${ }^{4}$ D. H. Johnson and D. E. Dudgeon, Array Signal Processing: Concepts and Techniques (Simon \& Schuster, 1992).
}

${ }^{5}$ F. Marinozzi, F. Bini, and A. Grandoni, "Experimental investigation of inter-element isolation in a medical array transducer at various manufacturing stages," Ultrson. Imaging 39, 62-74 (2017).

${ }^{6}$ X. L. Deán-Ben and D. Razansky, "On the link between the speckle free nature of optoacoustics and visibility of structures in limited-view tomography," Photoacoustics 4, 133-140 (2016).

${ }^{7}$ J. Xia and L. V. Wang, "Small-animal whole-body photoacoustic tomography: a review,” IEEE Trans. Biomed. Eng. 61, 1380-1389 (2014).

${ }^{8}$ J. J. Niederhauser, M. Jaeger, R. Lemor, P. Weber, and M. Frenz, "Combined ultrasound and optoacoustic system for real-time high-contrast vascular imaging in vivo," IEEE Trans. Med. Imaging 24, 436-440 (2005).

${ }^{9}$ R. G. Kolkman, P. J. Brands, W. Steenbergen, and T. G. van Leeuwen, "Real-time in vivo photoacoustic and ultrasound imaging," J. Biomed. Opt. 13, 050510 (2008).

${ }^{10}$ J. Yuan, G. Xu, Y. Yu, Y. Zhou, P. L. Carson, X. Wang, and X. Liu, "Real-time photoacoustic and ultrasound dual-modality imaging system facilitated with graphics processing unit and code parallel optimization," J. Biomed. Opt. 18, 086001 (2013).

${ }^{11}$ C.-W. Wei, T.-M. Nguyen, J. Xia, B. Arnal, E. Y. Wong, I. M. Pelivanov, and M. O'Donnell, "Real-time integrated photoacoustic and ultrasound (PAUS) imaging system to guide interventional procedures: Ex vivo study," IEEE Trans. Ultrason., Ferroelectr., Freq. Control 62, 319-328 (2015).

${ }^{12}$ A. Dima and V. Ntziachristos, "In-vivo handheld optoacoustic tomography of the human thyroid," Photoacoustics 4, 65-69 (2016).

${ }^{13}$ T. F. Fehm, X. L. Deán-Ben, and D. Razansky, "Four dimensional hybrid ultrasound and optoacoustic imaging via passive element optical excitation in a hand-held probe," Appl. Phys. Lett. 105, 173505 (2014).

${ }^{14}$ J. Jose, R. G. Willemink, W. Steenbergen, C. H. Slump, T. G. van Leeuwen, and S. Manohar, "Speed-of-sound compensated photoacoustic tomography for accurate imaging," Med. Phys. 39, 7262-7271 (2012).

${ }^{15}$ J. Xia, C. Huang, K. Maslov, M. A. Anastasio, and L. V. Wang, "Enhancement of photoacoustic tomography by ultrasonic computed tomography based on optical excitation of elements of a full-ring transducer array," Opt. Lett. 38, 3140-3143 (2013).

${ }^{16}$ E. Merčep, N. C. Burton, J. Claussen, and D. Razansky, "Whole-body live mouse imaging by hybrid reflection-mode ultrasound and optoacoustic tomography," Opt. Lett. 40, 4643-4646 (2015).

${ }^{17}$ E. Mercep, G. Jeng, S. Morscher, P.-C. Li, and D. Razansky, "Hybrid optoacoustic tomography and pulse-echo ultrasonography using concave arrays," IEEE Trans. Ultrason., Ferroelectr., Freq. Control 62, 1651-1661 (2015).

${ }^{18}$ American National Standards for the Safe Use of Lasers ANSI Z136.1 (American Laser Institute, 2000).

${ }^{19}$ M. Karaman, P.-C. Li, and M. O'Donnell, "Synthetic aperture imaging for small scale systems," IEEE Trans. Ultrason., Ferroelectr., Freq. Control 42, 429-442 (1995).

${ }^{20}$ M. Xu and L. V. Wang, "Universal back-projection algorithm for photoacoustic computed tomography,” Phys. Rev. E 71, 016706 (2005).

${ }^{21}$ L. Ding, X. L. Dean-Ben, C. Lutzweiler, D. Razansky, and V. Ntziachristos, "Efficient non-negative constrained model-based inversion in optoacoustic tomography," Phys. Med. Biol. 60, 6733-6750 (2015).

${ }^{22}$ M. P. Fronheiser, S. A. Ermilov, H.-P. Brecht, A. Conjusteau, R. Su, K. Mehta, and A. A. Oraevsky, "Real-time optoacoustic monitoring and three-dimensional mapping of a human arm vasculature," J. Biomed. Opt. 15, 021305 (2010).

${ }^{23}$ A. Taruttis, A. C. Timmermans, P. C. Wouters, M. Kacprowicz, G. M. van Dam, and V. Ntziachristos, "Optoacoustic imaging of human vasculature: Feasibility by using a handheld probe," Radiology 281, 256-263 (2016).

${ }^{24}$ M. Heijblom, D. Piras, F. M. van den Engh, M. van der Schaaf, J. M. Klaase, W. Steenbergen, and S. Manohar, "The state of the art in breast imaging using the Twente photoacoustic mammoscope: Results from 31 measurements on malignancies," Eur. Radiol. 26, 3874-3887 (2016).

${ }^{25}$ S. Y. Chuah, A. B. E. Attia, V. Long, C. J. H. Ho, P. Malempati, C. Y. Fu, S. J. Ford, J. S. S. Lee, W. P. Tan, D. Razansky, M. Olivo, and S. Thng, Skin Res. Technol. 23, 221-226 (2017).

${ }^{26}$ X. L. Deán-Ben, S. Gottschalk, B. McLarney, S. Shoham, and D. Razansky, "Advanced optoacoustic methods for multiscale imaging of in vivo dynamics," Chem. Soc. Rev. 46, 2158-2198 (2017).

${ }^{27}$ J. Aguirre, M. Schwarz, D. Soliman, A. Buehler, M. Omar, and V. Ntziachristos, "Broadband mesoscopic optoacoustic tomography reveals skin layers," Opt. Lett. 39, 6297-6300 (2014). 
${ }^{28}$ X. L. Deán-Ben, R. Ma, A. Rosenthal, V. Ntziachristos, and D. Razansky, "Weighted model-based optoacoustic reconstruction in acoustic scattering media," Phys. Med. Biol. 58, 5555 (2013).

${ }^{29}$ X. L. Deán-Ben, V. Ntziachristos, and D. Razansky, "Artefact reduction in optoacoustic tomographic imaging by estimating the distribution of acoustic scatterers," J. Biomed. Opt. 17, 110504 (2012).
${ }^{30}$ M. K. A. Singh and W. Steenbergen, "Photoacoustic-guided focused ultrasound (PAFUSion) for identifying reflection artifacts in photoacoustic imaging," Photoacoustics 3, 123-131 (2015).

${ }^{31}$ S. H. C. Ortiz, T. Chiu, and M. D. Fox, "Ultrasound image enhancement: A review," Biomed. Signal Process. Control 7, 419-428 (2012). 